\title{
DIP-MIP: Distributed Individual Paging Extension for Mobile IP in IP-Based Cellular Networks *
}

\author{
Chansophea Chuon ${ }^{\mathrm{a}, *}$ Sumanta Guha ${ }^{\mathrm{a}}$ \\ ${ }^{a}$ Computer Science and Information Management Program, School of Engineering \\ and Technology, Asian Institute of Technology, P.O. Box 4, Klong Luang, \\ Pathumthani, 12120, Thailand.
}

\begin{abstract}
The mobility-enabling protocol Mobile IP supports location registration but not paging. However, current cellular networks use registration as well as paging procedures to minimize signaling cost. Accordingly, an extension to Mobile IP using distributed individual paging, the so-called DIP-MIP, is proposed. In DIP-MIP, each mobile host derives its own paging area size by optimizing a signaling cost function based on its individual mobility pattern. The cost function itself may use either of two mobility models - fluid flow and random walk - and the performance of DIP-MIP is analyzed for both. The impact of various parameters on the DIP-MIP signaling cost is studied as well. The performance of DIP-MIP is shown to be superior to that of Mobile IP (MIP) in reducing signaling load, managing mobility and supporting a large number of mobile users in IP-based cellular networks.
\end{abstract}

Key words: IP-based cellular network, Mobile IP, mobility management, mobility model, paging.

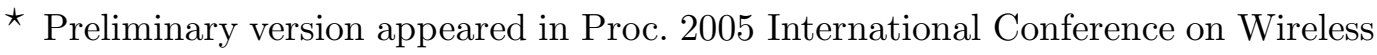
Networks, Communications and Mobile Computing (Wireless IP Symposium)

* Corresponding author.

Email addresses: Chansophea.Chuon@ait.ac.th (Chansophea Chuon), guha@ait.ac.th (Sumanta Guha).

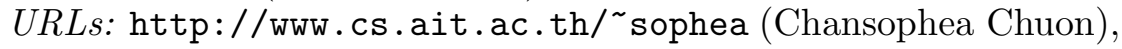
http://www.cs.ait.ac.th/ ${ }^{\sim}$ guha (Sumanta Guha).
} 


\section{Introduction}

The next generation of cellular networks will be integrated with IP technology to form so-called IP-based cellular networks. The drive is to deliver portfolio Internet services to mobile users. Mobile IP [8] is a mobility-enabling protocol that enables mobile hosts to maintain ongoing communication while moving from one subnet to another. Mobile IP introduces three new functional entities: the home agent (HA), foreign agent (FA), and mobile host (MH) (see [8] for details). Each $\mathrm{MH}$ is always identified by its home address, regardless of its point of attachment to the Internet. When an $\mathrm{MH}$ moves out of its home network it obtains a temporary address, called care-of address (CoA). This address is used to identify the $\mathrm{MH}$ in its currently visited network. When an MH moves from one subnet to another it registers its new CoA with its HA. Packets for the $\mathrm{MH}$ are sent with the home address of the $\mathrm{MH}$ as destination. The HA intercepts all IP packets destined for the MH and tunnels them to the serving FA of the MH. The FA decapsulates and forwards these packets to the MH. Mobile IP protocol requires that an $\mathrm{MH}$ registers its location to its home network whenever it enters a new subnet regardless of the $\mathrm{MH}$ state, active or idle, resulting in signaling overhead.

Mobile IP supports only location registration; however, IP layer paging is needed to reduce signaling overhead while the idle $\mathrm{MH}$ moves from one subnet to another and minimize the power consumption of the MHs. Various authors $[3-7,9,13,11,16]$ have proposed protocols to extend Mobile IP to support paging. An IP-based cellular network that supports IP layer paging protocols, typically, divides its coverage area into multiple paging areas. Paging is the procedure that allows the network to search for and locate the idle $\mathrm{MH}$ when there is a packet destined for the $\mathrm{MH}$, such that the $\mathrm{MH}$ does not need to register its precise location to the HA when moving within the paging area. An idle MH sends a location registration message to its HA only when it enters a new paging area, rather than when switching from one cell to another. A paging foreign agent (PFA) is a foreign agent that originates the paging request and is responsible for the paging process in its paging area. When the HA needs to contact an idle MH for packet delivery, the HA sends a request to the MH's currently serving PFA, and the PFA in turn pages the MH in its current paging area.

Ramjee et al [9] propose three different paging architectures where the paging agent function is implemented, respectively, on the home agent, domain root router, and the last visited foreign agent. The authors compare the performance of these schemes in terms of the paging load that can be supported and analyse the impact of varying the paging area size. However, a static paging area size is assumed for all MHs. 
Zhang et al [16] propose the Paging Extensions for Mobile IP protocol (PMIP). The analytic mobility model is based on fluid flow. Zhang et al assume parameters such as the MH's speed, incoming packet rate to be static and the same for all MHs. As a result the paging area size is aggregately configured for all MHs.

Castellucia [3] proposes an adaptive and individual paging scheme in which each $\mathrm{MH}$ computes its optimal paging area to extend Mobile IP to support paging. Incoming packet rate and mobility parameters are used in analysing signaling cost. Castellucia's scheme, though, requires frequent computation of the optimal paging area, which impacts power consumption of the MH. Further, a somewhat unrealistic assumption of the scheme, because it uses Euclidean distances in its mobility model, is that an MH has knowledge of its exact geographical location. Castellucia also calls for protocol modifications that are not compatible with the existing Mobile IP.

The paging area size is critical in networks that support paging: a high paging cost is generated when the paging area is large, while too small a paging area leads to excessive location registration cost. Optimal performance requires the system to balance these two cost components. Accordingly, we propose DIP-MIP, a distributed individual paging extension for Mobile IP in IP-based cellular networks. In DIP-MIP each MH has its own "view" of the network as divided into paging areas - this view being optimized, according to the MH's packet arrival rate and individual mobility pattern, to minimize signaling cost.

In DIP-MIP the paging traffic load is distributed to the FAs acting as PFAs in the network. Distribution of the PFA task increases robustness as well by reducing the system's sensitivity to the failure of individual PFAs. We show that DIP-MIP can provide significant savings in signaling bandwidth and help IP-based cellular networks to support a large number of MHs.

In Section II we describe DIP-MIP. In Section III we analyze the total signaling cost, comprised of location registration and paging cost, of DIP-MIP using two mobility models (fluid flow and random walk). In Section IV we compare the performance of DIP-MIP to the Mobile IP scheme. We conclude in Section V.

\section{DIP-MIP}

First, we present an overview of DIP-MIP and, then, detail location registration and paging procedures under DIP-MIP in IP-based cellular networks. 


\subsection{Overview}

Cellular networks use registration and paging procedures to minimize signaling overhead in the networks. Currently, Mobile IP does not support paging and a separate IP layer paging protocol is necessary to reduce signaling load over the limited wireless bandwidth, as well as the wired link. In the following we formalize DIP-MIP - an extension of Mobile IP with distributed individual paging.

An $\mathrm{MH}$ can be in one of two operational modes, active or idle. The active period includes the time that the $\mathrm{MH}$ is sending or receiving data together with an additional time interval determined by an internal timer. When the active period expires the $\mathrm{MH}$ enters idle mode. The MH's mode transition diagram is shown in Figure 1.

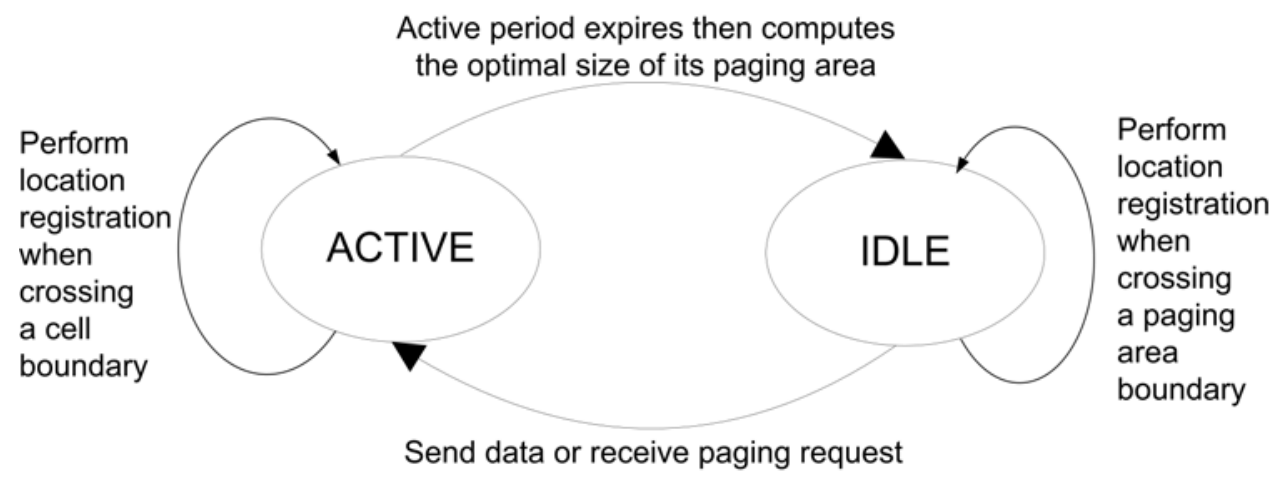

Fig. 1. An MH's mode transition diagram.

An MH accesses the Internet via IP-based cellular networks. An IP-based cellular network consists of cells and routers interconnected by wired link, while the networks themselves are connected to the Internet by gateway routers as shown in Figure 2.

Cells and routers are IP-based but cells have wireless communication with each MH. Each cell can act as both FA and PFA. A PFA is the cell located at the center of the paging area and is in charge of the paging process for that paging area. Consequently, if the $\mathrm{MH}$ is idle then the PFA is aware of this status. Routers provide routing services to MHs and cells provide location registration and paging services.

Cells are modelled as identical hexagons. Note that in IP-based cellular networks cells typically have different shapes and sizes - our assumption of homogeneity is to simplify analysis. Let $l$ be the perimeter length of a cell. Paging areas are configured as hexagonal shapes as well - each paging area is a sequence of concentric rings of cells - the number of rings is called the size of the paging area. Precisely, a center cell (cell acting as a PFA) is chosen and 


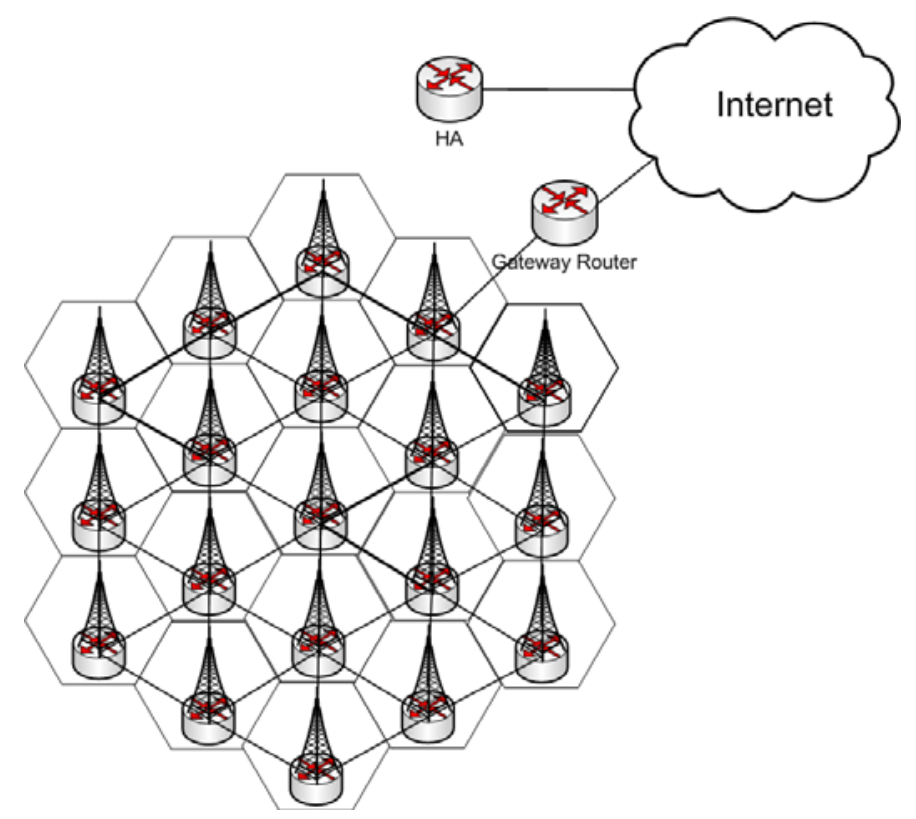

Fig. 2. An IP-based cellular network architecture.

labelled ' 1 ', each of the six cells of the next ring surrounding the center cell is labelled '2', each cell of the next ring is labelled ' 3 ', and so on till the label reaches the same value as the size of the paging area. Cells in a paging area are the nodes - and their adjacencies define the edges - of the paging area's mobility graph. See Figure 3.
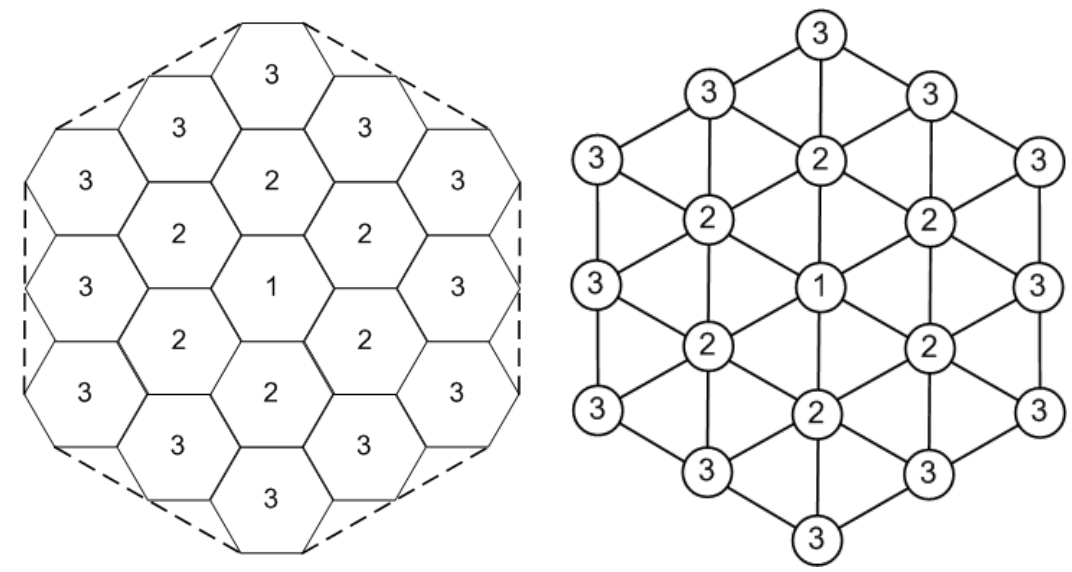

Fig. 3. Hexagonal paging area and mobility graph of size 3 .

In the paging area of size $K$, the ring labelled $k(k \geq 2)$ consists of $6(k-1)$ cells. Therefore, the number of cells in the paging area of size $K$ is therefore given as

$$
N(K)=1+6 \sum_{k=2}^{K}(k-1)=3 K(K-1)+1
$$


and the length of its perimeter is

$$
L(K)=(2 K-1) l
$$

and its area, called the coverage area, is

$$
\begin{aligned}
A(K) & =A(C) N(K) \\
& =\frac{\sqrt{3}}{24} l^{2}(3 K(K-1)+1)
\end{aligned}
$$

where $A(C)=\frac{\sqrt{3}}{24} l^{2}$ is the coverage area of a cell.

\subsection{Location Registration}

Location registration under DIP-MIP is as follows:

- In active mode DIP-MIP resembles Mobile IP: when an MH moves from a cell to another it performs location registration with its HA through its currently serving FA.

- When the MH transitions into idle mode, its currently serving FA becomes its PFA. At that time the $\mathrm{MH}$ derives its optimal paging area size $K^{\text {opt }}$ in a manner that we shall explain in Section 3.3. It registers its state and also requests the paging area of size $K^{\text {opt }}$ to the PFA and sends a location registration message to its $\mathrm{HA}$.

The MH keeps a buffer of size $3 K^{\text {opt }}\left(K^{\text {opt }}-1\right)+1$ to store the IDs of cells sent from the serving PFA within the visited paging area so that it can determine when it is exiting the paging area. The $\mathrm{MH}$ does not need to send further location registration messages to its HA when traveling within the paging area, which reduces signaling traffic, as well as power consumption of the MH.

In idle mode, when the $\mathrm{MH}$ moves out of its current paging area, it registers with the new FA, which then serves as its PFA. The MH requests the paigng area of size $K^{o p t}$ from the new PFA. Then it deletes the cell IDs saved in the previous buffer and stores the cell IDs sent by the new PFA. The MH sends a location registration message to its HA as well.

Note that the $\mathrm{MH}$ recomputes $K^{\text {opt }}$ only when it transitions from active to idle. 


\subsection{Paging}

Incoming packets for the $\mathrm{MH}$ are intercepted by the HA and tunnelled to the registered FA if the $\mathrm{MH}$ is in active mode, or the serving PFA if the $\mathrm{MH}$ is in idle mode. Subsequently, if the $\mathrm{MH}$ is in active mode, the packets will be forwarded directly to the $\mathrm{MH}$ from the currently serving FA. In case the $\mathrm{MH}$ is in idle mode, the PFA buffers the packets and broadcasts a paging request for the $\mathrm{MH}$ within the paging area.

After receiving the request the $\mathrm{MH}$ changes its status to active and sends a location registration message to its HA through its currently serving FA. It also registers its current location with its previous PFA. The previous PFA then sends the buffered packets to the $\mathrm{MH}$ via its currently serving FA. Subsequent packets are sent directly to the MH's currently serving FA.

\section{DIP-MIP Signaling Cost Analysis}

In this section we analyze the signaling cost of DIP-MIP which is composed of two components: location registration cost and paging cost. To strengthen the signaling cost analysis, we apply both mobility models commonly used in the literature: fluid flow [12], [16] and random walk [1], [2]. The fluid-flow model is appropriate for users with high mobility, but infrequent speed and direction changes. The random walk model, on the other hand, is appropriate for users whose mobility is confined to a limited geographical area such as one building.

We define the following parameters:

- $\lambda$ is the packet arrival rate, which is also the paging rate.

- $\theta$ is the ratio of idle time to active time of the MH.

- $C_{w}$ is the location registration cost per hop over the wired link.

- $C_{l}$ is the location registration cost over a cell wireless link, which is assumed to be identical with the paging cost for an incoming packet over a cell wireless link.

- $D_{F P}$ is the distance in hops between its currently serving FA and the PFA.

- $D_{F H}$ is the distance in hops between its currently serving FA and the HA.

The cost of location registration is proportional to the distance between the $\mathrm{MH}$ and its mobility agents to which it is sending location registration messages. Therefore, the cost of a location registration of an $\mathrm{MH}$ to its PFA and HA are given by

$$
C_{P F A}=D_{F P} C_{w}+C_{l}
$$




$$
C_{H A}=D_{F H} C_{w}+C_{l}
$$

The cost of paging in a paging area is equal to the total cost of sending signaling messages from within each cell in the paging area. The cost of paging in a paging area of size $K$ is, therefore, given by

$$
\begin{aligned}
C_{P}(K) & =C_{l} N(K) \\
& =C_{l}(3 K(K-1)+1)
\end{aligned}
$$

\subsection{Fluid Flow Model}

In the fluid flow model the direction of the $\mathrm{MH}$ in a cell or paging area is uniformly distributed in $[0,2 \pi]$. Let $\rho$ denote the density of MHs per unit area. Let $V$ be the average velocity of MHs. The average number of MHs crossing a cell boundary per unit time is given by

$$
R(C)=\frac{\rho V l}{\pi}
$$

and the average number crossing the boundary of a paging area of size $K$ per unit time is given by

$$
\begin{aligned}
R(K) & =\frac{\rho V L(K)}{\pi} \\
& =\frac{\rho V(2 K-1) l}{\pi}
\end{aligned}
$$

The average location registration rate $U(K)$ per $\mathrm{MH}$, from a paging area of size $K$, is given by

$$
\begin{aligned}
U(K) & =\frac{R(K)}{\rho A(K)} \\
& =\frac{8 \sqrt{3}}{\pi l} V\left(\frac{2 K-1}{3 K(K-1)+1}\right)
\end{aligned}
$$

The signaling cost (sum of location registration cost and paging cost) per $\mathrm{MH}$ and per unit time is the following:

$$
C_{F F}(K, \lambda, V)=\theta \lambda C_{P}(K)+\theta \lambda\left(C_{P F A}+2 C_{H A}\right)+2 U(K) C_{H A}
$$

As a result we get 


$$
\begin{aligned}
C_{F F}(K, \lambda, V)= & \theta \lambda(3 K(K-1)+1) C_{l}+\theta \lambda\left(C_{P F A}+2 C_{H A}\right)+ \\
& \frac{16 \sqrt{3}}{\pi l} V\left(\frac{2 K-1}{3 K(K-1)+1}\right) C_{H A}
\end{aligned}
$$

where the first term is the paging cost that occurs at every incoming packet, the second term is the cost of location registration to the PFA and the HA after a paging request, while the last term represents the cost of location registration to the $\mathrm{HA}$ when the $\mathrm{MH}$ exits its current paging area. Note that the cost of location registration to the serving HA is multiplied by 2 because the location registration to the HA needs to be replied.

\subsection{Random Walk Model}

In case of the random walk model we assume the following:

(a) The probability that an MH remains in the same cell in the next unit of time is $q$.

(b) If the $\mathrm{MH}$ exits a cell it goes to each adjacent cell with equal probability $\frac{1}{6}$.

Given the paging area configuration as depicted in Figure 3, the transition probability that an $\mathrm{MH}$ currently located in ring $k$ travels to ring $k+1$ in increase of distance from the PFA cell is

$$
\alpha_{k}^{+}= \begin{cases}(1-q), & \text { if } k=1 \\ (1-q)\left(\frac{1}{3}+\frac{1}{6(k-1)}\right), & \text { if } 2 \leq k \leq K\end{cases}
$$

and that it travels to ring $k-1$ in decrease of distance from the PFA cell is

$$
\alpha_{k}^{-}= \begin{cases}0, & \text { if } k=1 \\ (1-q)\left(\frac{1}{3}-\frac{1}{6(k-1)}\right), & \text { if } 2 \leq k \leq K\end{cases}
$$

We define a Markov chain [10] as follows: state $k(k \geq 1)$ corresponds to the ring of index $k$. The $\mathrm{MH}$ is said to be in state $k$ if it currently resides in the ring of index $k$. A state transition diagram for the Markov chain of a paging area of size $K$ is shown in Figure 4 . We denote by $\delta_{k, K}$ the steady-state probability of state $k$ within a paging area composed of $K$ rings. Based on the transition probabilities shown in Equations (11) and (12), $\delta_{k, K}$ can be expressed in terms 

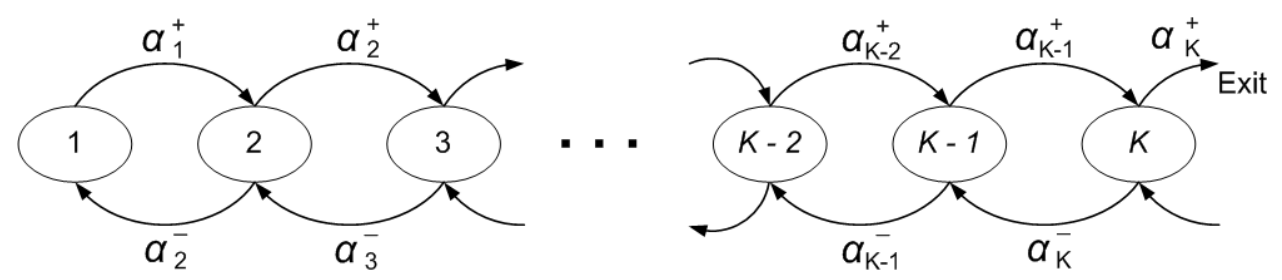

Fig. 4. State diagram of a Markov chain with paging area of size $K$.

of the steady-state probability of the center cell $\delta_{1, K}$ as

$$
\delta_{k, K}=\delta_{1, K} \prod_{i=1}^{k-1} \frac{\alpha_{i}^{+}}{\alpha_{i+1}^{-}}, \text {for } 2 \leq k \leq K
$$

With the requirement

$$
\sum_{k=1}^{K} \delta_{k, K}=1
$$

We can write

$$
\delta_{1, K}=\frac{1}{1+\sum_{k=2}^{K} \prod_{i=1}^{k-1} \frac{\alpha_{i}^{+}}{\alpha_{i+1}^{-}}}
$$

According to this mobility model, if an MH moves from ring $K$ to ring $K+1$, then it performs a location registration to its HA. The probability that the $\mathrm{MH}$ performs a location registration is, therefore, given as the following:

$$
\delta_{K, K} \alpha_{K}^{+}
$$

Let $T$ be the average cell residence time of an MH. Then, the signaling cost per unit time of an $\mathrm{MH}$ is given by

$$
\begin{gathered}
C_{R W}(K, \lambda, T)=\frac{1}{T}\left(\theta \lambda(3 K(K-1)+1) C_{l}+\theta \lambda\left(C_{P F A}+2 C_{H A}\right)+\right. \\
\left.2 \delta_{K, K} \alpha_{K}^{+} C_{H A}\right)
\end{gathered}
$$

As a result we get

$$
\begin{aligned}
C_{R W}(K, \lambda, T)= & \frac{1}{T}\left(\theta \lambda(3 K(K-1)+1) C_{l}+\theta \lambda\left(C_{P F A}+2 C_{H A}\right)+\right. \\
& \left.2(1-q)\left(\frac{2 K-1}{3 K(K-1)+1}\right) C_{H A}\right)
\end{aligned}
$$




\subsection{Computing Optimal Paging Area Size}

The optimal paging area of size $K^{o p t}$, for each $\mathrm{MH}$, is defined as the value $K$ that minimizes the signaling cost functions in Equations (10) and (18). As $K$ is integral, we cannot derive with respect to $K$ to find a minimum; instead, we follow the method of [12] to derive the optimal paging area size. We define $\Delta_{F F}$ and $\Delta_{R W}$ as the cost difference functions between the paging area of size $K$ and the paging area of size $K-1(K \geq 2)$ in the fluid flow and random walk models, respectively, i.e.,

$$
\begin{gathered}
\Delta_{F F}(K, \lambda, V)=C_{F F}(K, \lambda, V)-C_{F F}(K-1, \lambda, V) \\
\Delta_{R W}(K, \lambda, T)=C_{R W}(K, \lambda, T)-C_{R W}(K-1, \lambda, T)
\end{gathered}
$$

where $\lambda$ is the incoming packet rate, $V$ is the average velocity of each $\mathrm{MH}$ in fluid flow model and $T$ is the cell average residence time of each $\mathrm{MH}$ in random walk mobility model. Given $\Delta_{F F}$ and $\Delta_{R W}$, the equations to find the optimal size of the paging area in either model are the following:

$$
\begin{aligned}
& K_{F F}^{\text {opt }}(\lambda, V)= \\
& \begin{cases}1, & \Delta_{F F}(2, \lambda, V)>0 \\
\max \left\{K: \Delta_{F F}(K, \lambda, V) \leq 0\right\} & \text { otherwise }\end{cases} \\
& K_{R W}^{\text {opt }}(\lambda, T)= \\
& \begin{cases}1, & \Delta_{R W}(2, \lambda, T)>0 \\
\max \left\{K: \Delta_{R W}(K, \lambda, T) \leq 0\right\} & \text { otherwise }\end{cases}
\end{aligned}
$$

From these equations, the optimal paging area size - both $K_{F F}^{o p t}$ and $K_{R W}^{o p t}-$ depends upon the packet arrival rate $\lambda$ and the average velocity $V$ under the fluid flow model, and the average cell residence time $T$ under the random walk model.

\subsection{Performance Analysis}

We now analyze the DIP-MIP signaling cost which is comprised of two components: registration cost and paging cost. Our evaluation considers both under 
the fluid flow, as well as random walk, mobility models. Parameter values used in this evaluation are mostly adopted from [14] and [16] . They are shown in Table 1.

Table 1

Performance Analysis Parameters

\begin{tabular}{|c|c|}
\hline Parameter & Value \\
\hline$l$ & $400 \mathrm{~m}$ \\
$V$ & $8.9 \mathrm{~m} / \mathrm{s}$ \\
$T$ & $20 \mathrm{~s}$ \\
$q$ & 0.4 \\
$\theta$ & 0.5 \\
$C_{w}$ & 0.5 \\
$C_{l}$ & 1.0 \\
$D_{F P}$ & 10 hops \\
$D_{F H}$ & 25 hops \\
\hline
\end{tabular}

Firstly, the impact of the paging area size $K$ on the signaling cost of DIP-MIP is observed under various packet arrival rates $(\lambda=0.4, \lambda=0.8$ and $\lambda=2.0)$. Figure 5 plots the signaling cost as a function of $K$ under the fluid flow model, while Figure 6 does likewise for the random walk model. As is seen in Figures 5 and 6 , the signaling costs of DIP-MIP increases as the size of the paging area increases. The signaling cost is the highest under $\lambda=2.0$. Intuitively, the reason is that, when there is a large number of cells in the paging area (big paging area), the cost of paging increases while that of location registration falls. Hence, the paging cost dominates the location registration costs.

It is seen that the paging area size $K$ is critical to minimizing signaling cost. In DIP-MIP, this is achieved as the optimal size of the paging area, derived by each $\mathrm{MH}$ based on its own individual parameters. It is observed that when the incoming packet rate is high, the paging area size $K$ must be small for DIP-MIP to reach optimal performance; conversely, when the incoming packet rate is low, the paging area size $K$ should be large. In DIP-MIP, an MH sends a location registration to the serving $\mathrm{HA}$ only when the $\mathrm{MH}$ crosses the paging area boundary, or there is an incoming packet for the MH. Therefore, DIP-MIP significantly reduces signaling cost in comparison to Mobile IP, which requires the $\mathrm{MH}$ to send location registration whenever it crosses a cell boundary. 


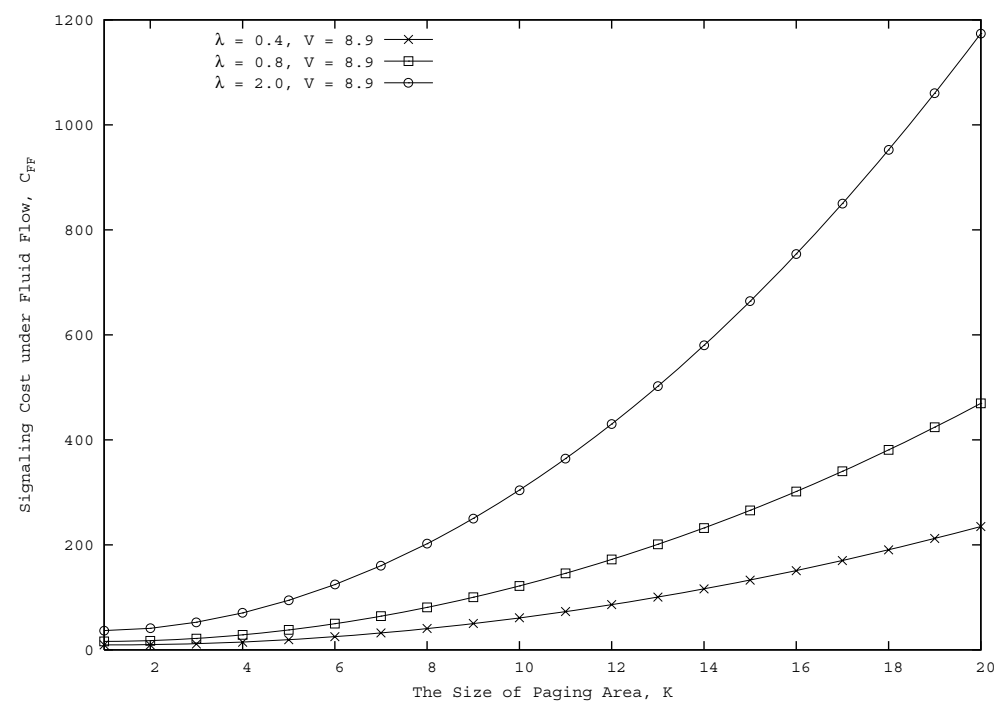

Fig. 5. Signaling cost as a function of paging area size $K$ under fluid flow.

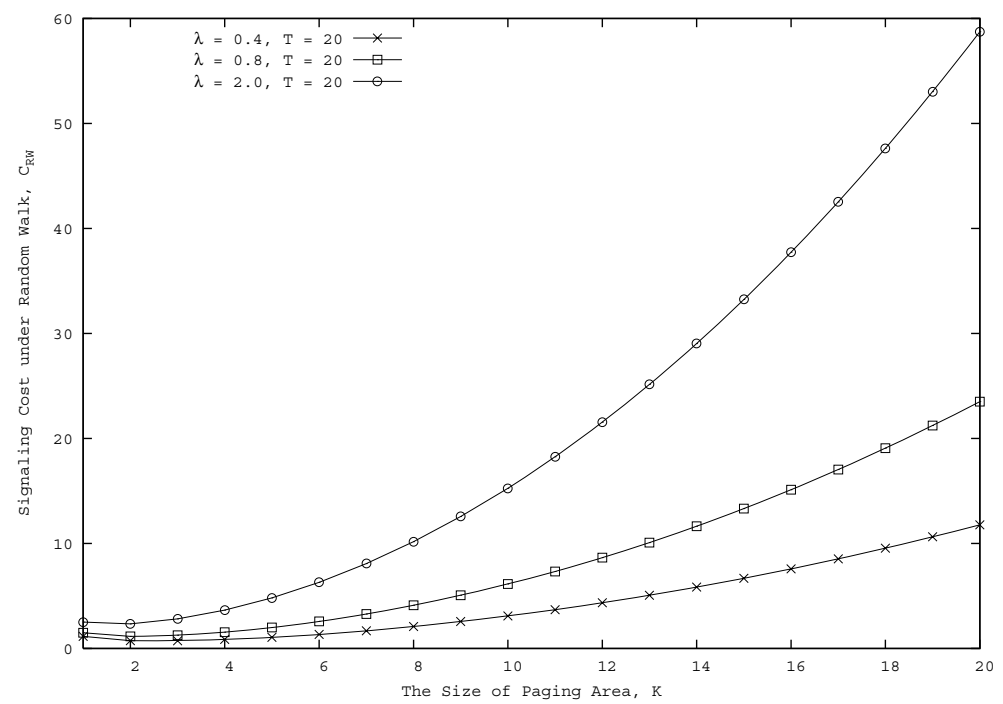

Fig. 6. Signaling cost as a function of paging area size $K$ under random walk.

Next, Figures 7 and 8 show, respectively, the signaling cost as a function of the MH's average velocity $V$ under the fluid-flow model, and average residence time $T$ under the random walk mobility model. The paging area size $K$ is set to 2,4 and 6 , successively, and the incoming packet rate $\lambda=0.4$.

Under the fluid flow model, the higher the average velocity of an $\mathrm{MH}$, the higher its rate of crossing the paging area boundary, and, consequently, the higher the location registration cost that it generates. With the paging cost remains unchanged as the velocity increases, the signaling cost in DIP-MIP, as shown in Figure 7, increases slowly because the $\mathrm{MH}$ is required to send a location registration only when it crosses a paging area boundary or receives incoming packets. Intuitively, the reason is that, when the mobility rate is 
high, the cost of location registration dominates. DIP-MIP still significantly reduces the location registration traffic compared to Mobile IP, which requires the $\mathrm{MH}$ to send location registration whenever it crosses a cell boundary.

Figure 8 shows the signaling cost as a function of the average cell residence time $T$ in the random walk model. The probability that the $\mathrm{MH}$ remains in the current cell at the next time unit $q=0.4$. The MH performs fewer movements as the average cell residence time increases resulting in lower location registration cost. DIP-MIP signaling cost decreases as the the cell residence time increases. As $T$ increases, DIP-MIP achieves optimal performance when the MH's paging area size is small.

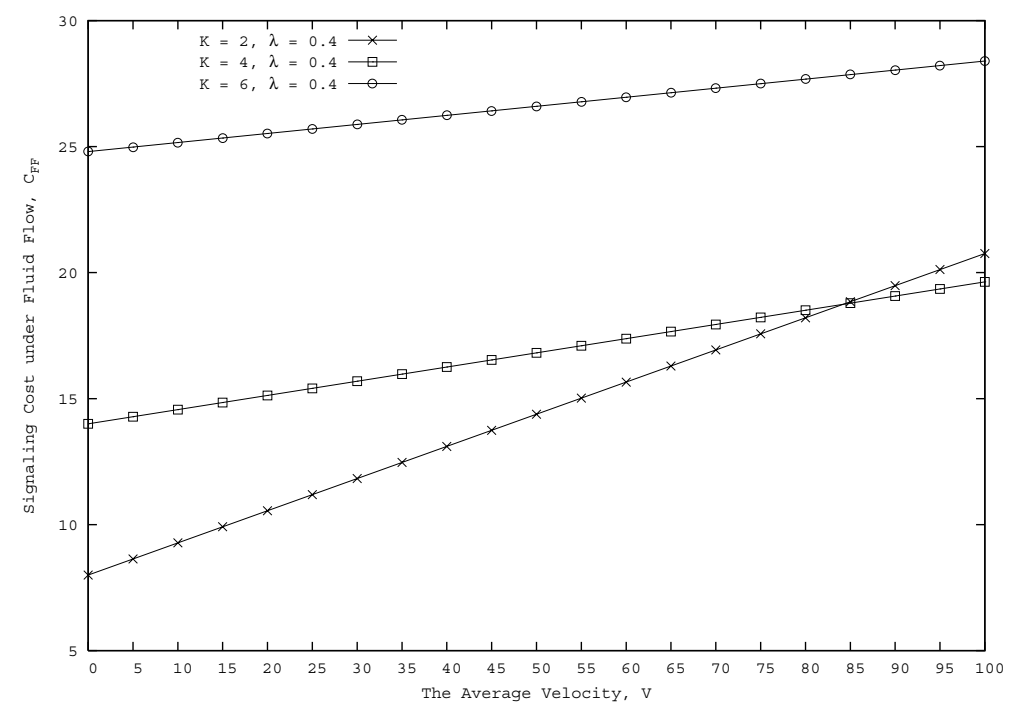

Fig. 7. Signaling cost as a function of the average velocity $V$ under fluid flow.

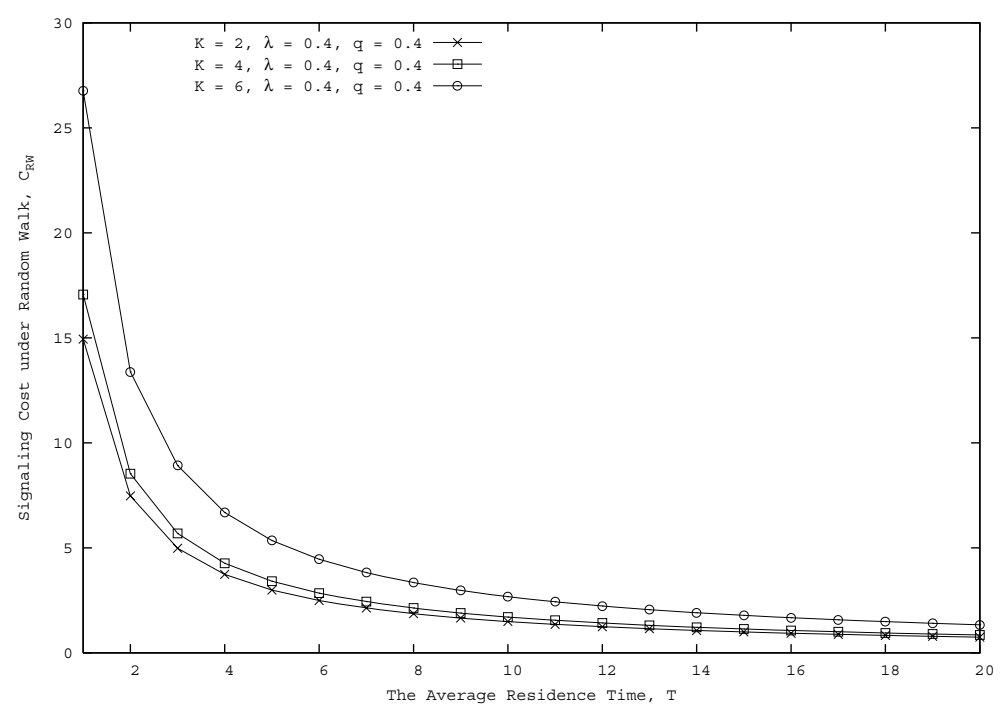

Fig. 8. Signaling cost as a function of the average residence time $T$ under random walk. 
Next, we see how the signaling cost changes with the packet arrival rate $\lambda$, given paging areas of sizes 2, 4 and 6, respectively. As is seen in Figures 9 and 10 , the signaling cost is the highest when $K=6$. This is because when the paging area size $K$ is large, the paging cost is high. The signaling cost of DIP-MIP increases linearly with incoming packet rate. When the packet arrival rate is large, the paging cost increases and dominates the cost of location registration, which subsequently results in a high signaling cost. In case the incoming packets rate $\lambda$ is large, the optimal performance of DIP-MIP is reached when the $\mathrm{MH}$ has a small paging area. As is seen from the discussion in Section 3.3, the incoming packet rate is critical to determining the optimal size of the page area.

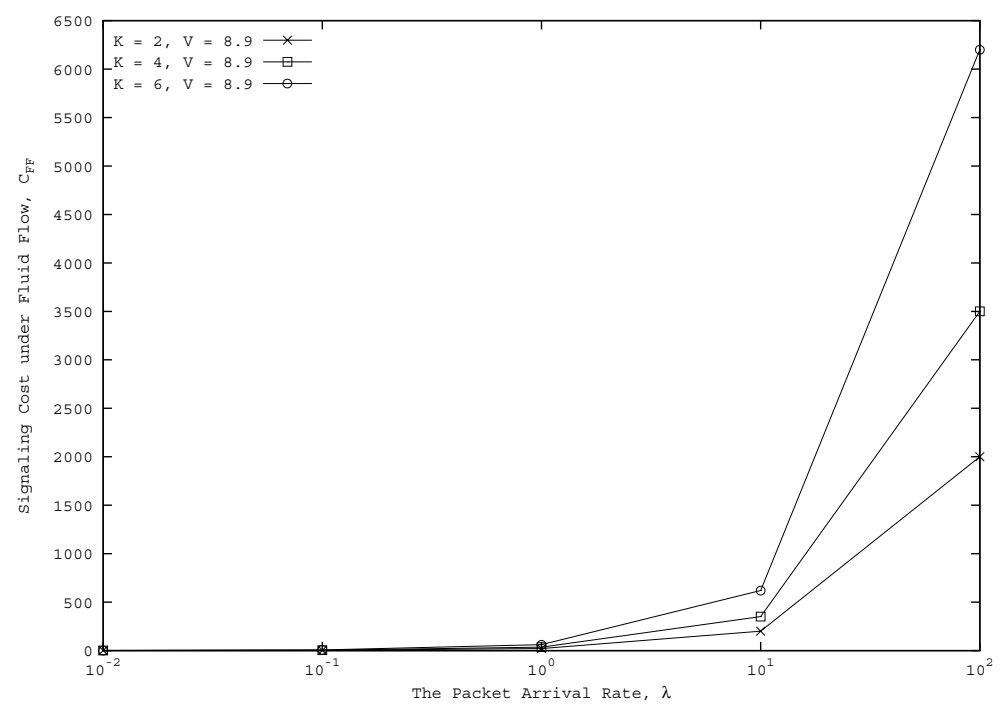

Fig. 9. Signaling cost as a function of the packet arrival rate $\lambda$ under fluid flow.

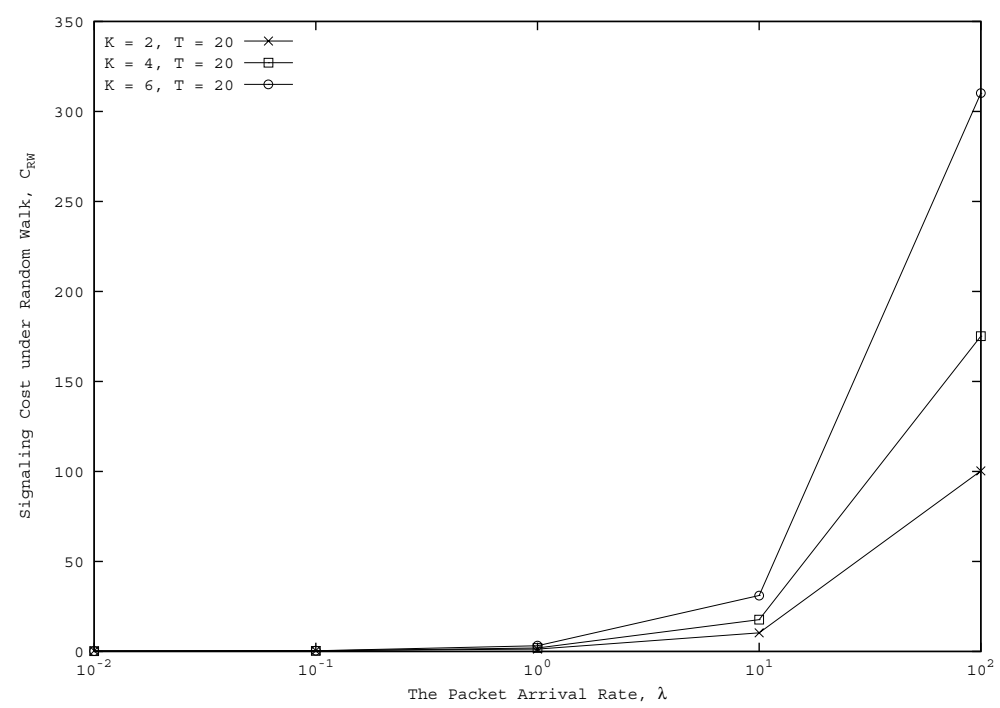

Fig. 10. Signaling cost as a function of the packet arrival rate $\lambda$ under random walk. 
Figure 11 and 10 plot the optimal size of paging area $K^{\text {opt }}$ as functions of the packet arrival rate $\lambda$. The average velocity $V=8.9$ and the average cell residence time $T=20$, under fluid flow and random walk mobility models respectively. It is seen that under both models, the optimal size of the paging area $K^{\text {opt }}$ decreases as the packet arrival rate $\lambda$ increases. This is obvious that, when the incoming packet rate is high - the paging cost dominates the registration cost - the optimal size of the paging area must be small for DIPMIP to reach optimal performance; conversely when the packet arrival rate $\lambda$ is low, the size of the paging area should be large in order to achieve the optimal performance. It is clear that DIP-MIP scheme significantly saves the signaling bandwidth because each $\mathrm{MH}$ computes and uses its optimal size of the paging area based on its individual packet arrival rate and mobility patterns.

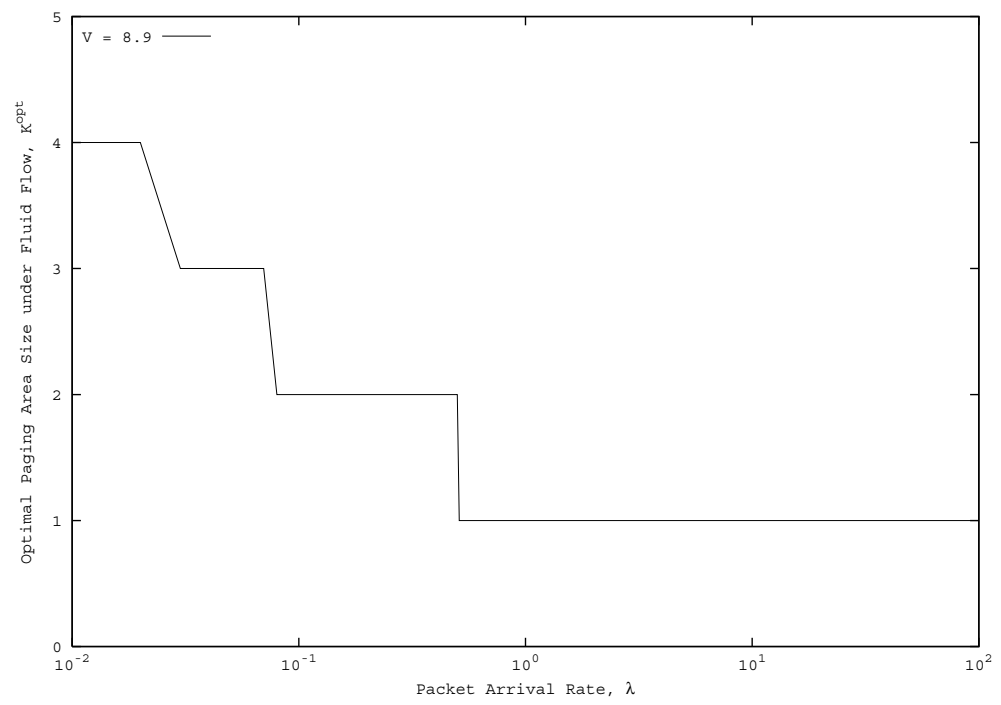

Fig. 11. Optimal paging area size as a function of the packet arrival rate $\lambda$ under fluid flow.

In DIP-MIP, the signaling cost is sensitive to the ratio $\theta$ of idle time to active time. When $\theta=0$ (MHs are active all the time), the DIP-MIP scheme resembles that of Mobile IP. However, DIP-MIP begins to outperform Mobile IP as the ratio $\theta$ increases from 0 (in this context, note that, typically, an $\mathrm{MH}$ is idle more often than it is active).

\section{MIP Versus DIP-MIP: Performance Analysis}

In the MIP scheme there is no notion of paging. The total signaling cost is the cost of location registration only. To update its location, an $\mathrm{MH}$ sends a registration message to its HA when crossing a cell boundary. To make the comparison with DIP-MIP, we study the signaling cost of MIP under the paging area of size $K$ using the parameters shown in Table 1. We also 


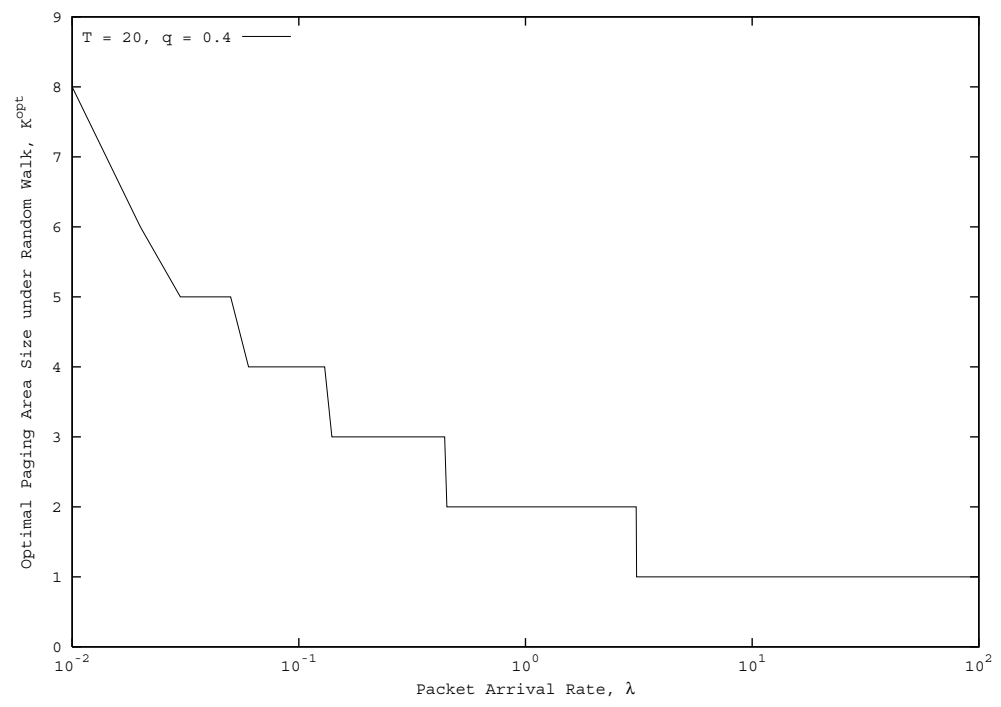

Fig. 12. Optimal paging area size as a function of the packet arrival rate $\lambda$ under random walk.

demonstrate some numerical results from the comparison. The signaling cost of MIP under both fluid flow $\left(C_{M I P-F F}\right)$ and random walk $\left(C_{M I P-R W}\right)$ mobility models can be derived as the following:

$$
\begin{aligned}
& C_{M I P-F F}(K, V)=\frac{16 \sqrt{3}}{\pi l} V(3 K(K-1)+1) C_{H A} \\
& C_{M I P-R W}(K, T)=\frac{2}{T}(1-q)(3 K(K-1)+1) C_{H A}
\end{aligned}
$$

Firstly, the impact of the paging area of size $K$ on the signaling costs of MIP and DIP-MIP is investigated under the packet arrival rate $\lambda=2$. Figures 13 and 14, respectively, show the signaling cost as a function of $K$ under the fluid flow and random walk models. Both the signaling cost of MIP and DIP-MIP increase as the size of the paging area increases. As shown in Figure 13, when the size of the paging area is small $(K \leq 3)$ the signaling cost of DIP-MIP is a little higher than MIP. The reason is that when the paging area is small DIP-MIP's sensitivity to the packet arrival rate predominates (MIP, on the other hand, is not sensitive to the packet arrival rate). In the DIP-MIP scheme the higher the packet arrival rate the greater the paging cost generated.

When the number of cells in the paging area increases, the signaling cost of MIP increases faster than DIP-MIP. This is clear because in MIP the MH is required to send a location registration message whenever it crosses a cell boundary. 
Figure 14 shows likewise for the random walk model. As the size of the paging area increases, the signaling cost of MIP increases sharply and is much more higher than DIP-MIP. As is seen from the figures and analysis, DIP-MIP can significantly reduce the signaling cost.

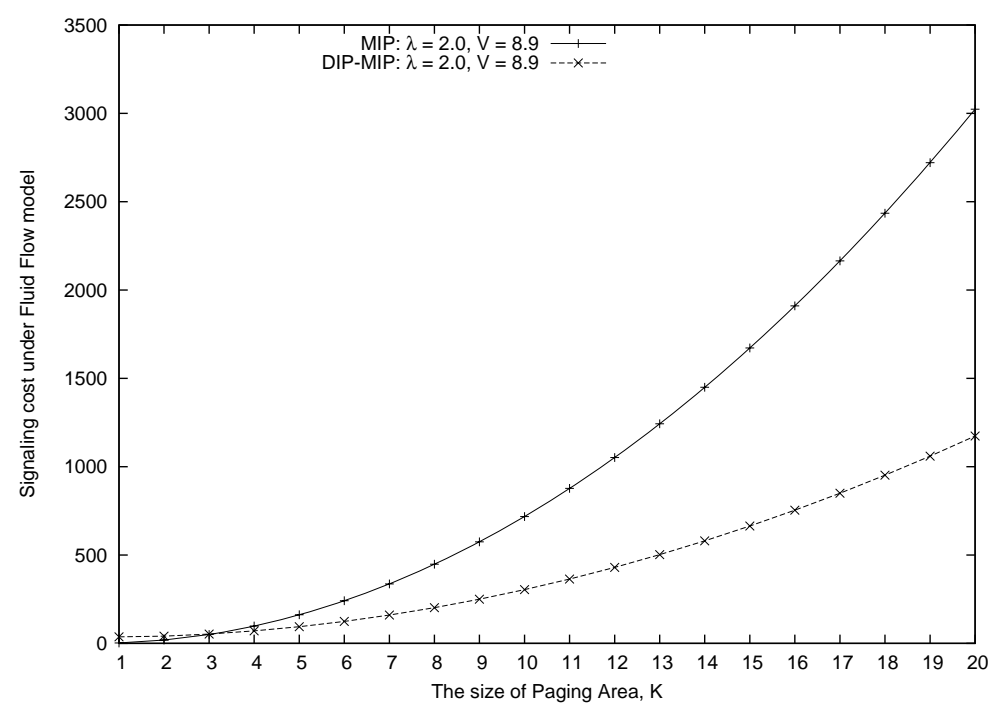

Fig. 13. MIP and DIP-MIP signaling cost as a function of paging area size $K$ under fluid flow.

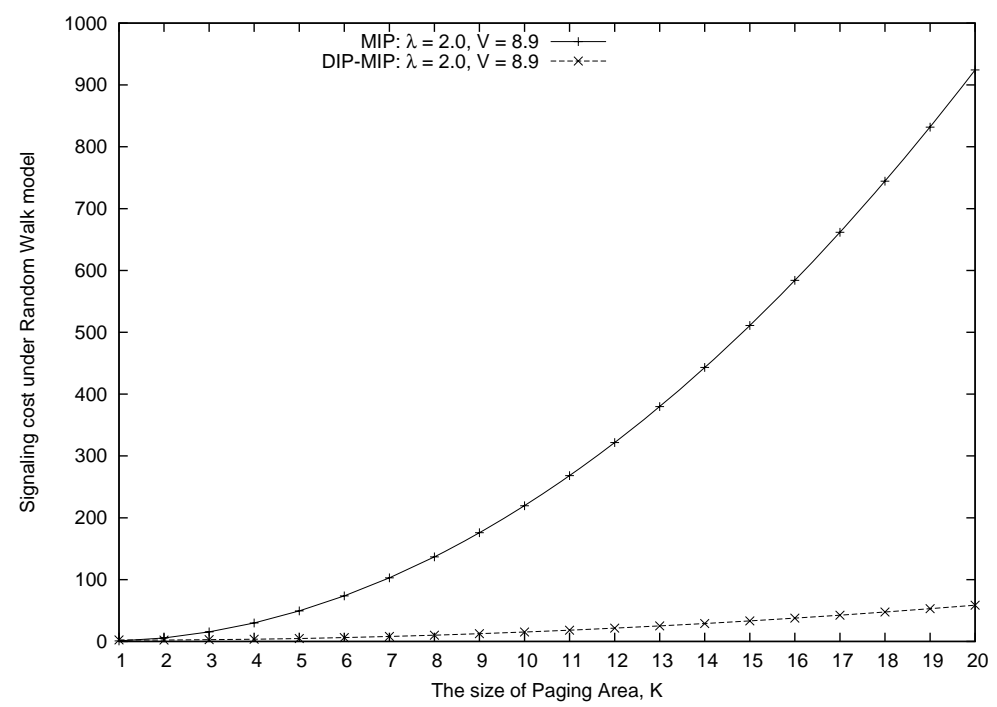

Fig. 14. MIP and DIP-MIP signaling cost as a function of paging area size $K$ under random walk. 
Figures 15 and 16 plot, respectively, the signaling cost of MIP and DIP-MIP as the functions of the MH's average velocity $V$ under the fluid flow model and the average cell residence time $T$ under the random walk model. The size of the paging area $K=2$ and the packet arrival rate $\lambda$ is set to 2 . Under the fluid flow model, the signaling cost of MIP is particularly sensitive to the average velocity of the MH. The higher the average velocity of the $\mathrm{MH}$, the higher its rate of crossing cell boundaries. Consequently, it generates higher location registration cost. As is shown in Figure 15, the signaling cost of MIP increases rapidly while that of DIP-MIP hardly changes. This is a consequence of the fact in DIP-MIP an MH is required to update its location only whenever it crosses a paging area boundary or if there is an incoming packet. As the velocity of the MH increases, DIP-MIP outperforms MIP. Figure 16 shows the signaling cost of both schemes as a function of the average cell residence time $T$ in the random walk model. Both MIP and DIP-MIP signaling costs decrease as the cell residence time increases. However, DIP-MIP is still better than MIP in terms of performance and reduction of signaling overhead in an IP-based cellular network.

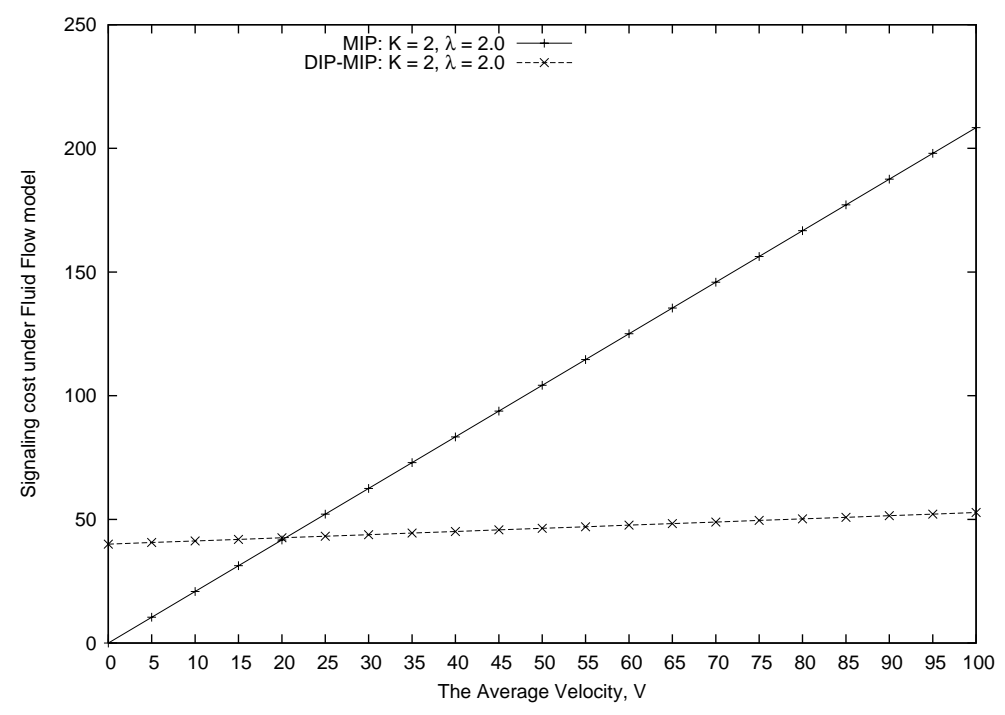

Fig. 15. MIP and DIP-MIP signaling cost as a function of the average velocity $V$ under fluid flow.

\section{Conclusion}

We have introduced a simple distributed individual paging extension to Mobile IP protocol for mobility management in IP-based cellular networks, called DIP-MIP. In the proposed scheme each MH computes its optimal paging area size according to its packet arrival rate and mobility parameters to minimize signaling load. Further, DIP-MIP is backward compatible and can be used with the existing Mobile IP protocol given a minimal set of paging extension. 


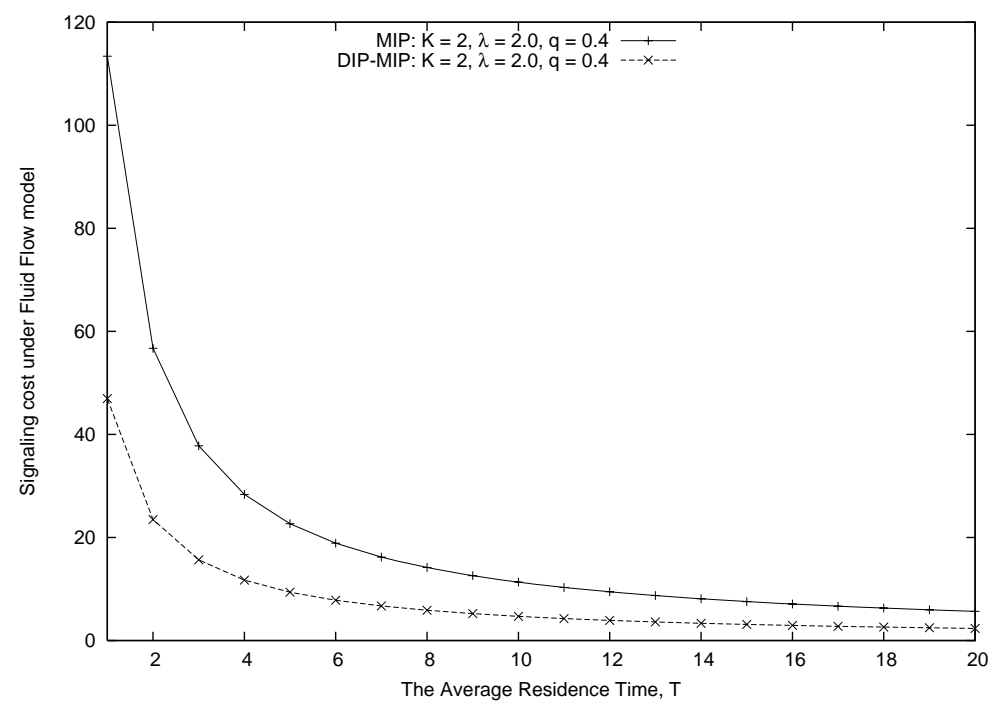

Fig. 16. MIP and DIP-MIP signaling cost as a function of the average residence time $T$ under random walk.

The signaling cost of DIP-MIP is analysed using the fluid flow and random walk mobility models. Analysis of DIP-MIP indicates that the paging area size and incoming packet rate are critical factors in minimizing the signaling cost. DIP-MIP has been shown to significantly save signaling cost and miminize the power consumption at the MHs in comparison to Mobile IP. DIP-MIP is robust, scalable and easily deployed to mobility management in IP-based cellular networks. In future work, we shall consider augmenting DIP-MIP with methods to vary shapes of individual paging areas, which we suspect will further enhance its performance. To achieve a more realistic performance comparison, we plan to use the Network Simulator (NS2) [15] to model DIP-MIP.

\section{References}

[1] I. F. Akyildiz, J.S.M Ho, A Mobile User Location Update and Paging Under Delay Constraints Proceedings ACM SIGCOMM, 1995, 244-255.

[2] I. F. Akyildiz, W. Wang, A Dynamic Location Management Scheme for NextGeneration Multitier PCS Systems. Proceedings IEEE Transactions on Wireless Communications, Vol. 1, No. 1, 2002, 178-189.

[3] C. Castellucia, Extending Mobile IP with Adaptive Individual Paging: A Performance Analysis Proceedings ACM Mobile Computing and Communication Review, Vol. 5, No. 2, 1999, 15-26.

[4] T. Choi, L. Kim, J. Nah, J. Song, Combinatorial mobile IP: a new efficient mobility management using minimized paging and local registration in mobile IP environments, ACM Wireless Networks, Vol. 10, No. 3, 2004, 311-321. 
[5] H.T. Do, Y. Onozato, Merit of Adaptability to Mobile User Parameters in IP Paging, Proceeding Second Annual Conference on Wireless On-demand Network Systems and Services (WONS'05), 2005, 246-251.

[6] J. Kempf, Dormant Mode Host Alerting (IP Paging) Problem Statement, RFC 3132, 2001.

[7] J. Kempf, C. Castellucia, P. Mutaf, N. Nakajima, Y. Ohba, R. Ramjee, Y. Saifullah, B. Sarikaya, X. Xu, Requirements and Functional Architecture for an IP Host Alerting Protocol, RFC 3154, 2001.

[8] C. Perkins, IP Mobility Support for IPv4, RFC $3344,2002$.

[9] R. Ramjee, L. Li, T. L. Porta and S. Kasera, IP Paging Service for Mobile Hosts, Wireless Networks, Vol. 8, No. 5, 2001, 427-441.

[10] S.M. Ross, Introduction to Probability Models, Amsterdam, Academic Press, 2003.

[11] A.G. Valkó, Cellular IP: a new approach to Internet host mobility, $A C M$ SIGCOMM Computer Communication Review ,

Vol. 29, No. 1, 1999, 50-65.

[12] H. Xie, S. Tabbane and D.J. Goodman, Dynamic Location Area Management and Performance Analysis Proceedings IEEE VTC, 1993, 536-539.

[13] J. Xie, User independent paging scheme for mobile IP, Wireless Networks, Vol. 12, No. 2, 2006, 145-158.

[14] J. Xie, I.F. Akyildiz, A Distributed Dynamic Regional Location Management Scheme for Mobile IP, Proceedings IEEE INFOCOMM, 2002, 1069-1078.

[15] UCB/LBLN/VINT Network Simulator (NS), http://www.isi.edu/nsnam.

[16] X. Zhang, J. G. Castellanos and A. T. Campbell, P-MIP: Paging Extension for Mobile IP, Proceedings ACM Mobile Networks and Applications, 2002, 127-141. 